\title{
Lecture et langage en français langue seconde : problèmes cognitifs et didactiques
}

\section{Michèle Verdelhan-Bourgade}

\section{(2) OpenEdition \\ 1 Journals}

Édition électronique

URL : http://journals.openedition.org/trema/2180

DOI : $10.4000 /$ trema. 2180

ISSN : 2107-0997

\section{Éditeur}

Faculté d'Éducation de l'université de Montpellier

\section{Édition imprimée}

Date de publication : 1 juin 1995

Pagination : 38-46

ISSN : 1167-315X

\section{Référence électronique}

Michèle Verdelhan-Bourgade, « Lecture et langage en français langue seconde : problèmes cognitifs et didactiques », Tréma [En ligne], 7 | 1995, mis en ligne le 23 septembre 2013, consulté le 19 avril 2019. URL : http://journals.openedition.org/trema/2180 ; DOI : 10.4000/trema.2180

Ce document a été généré automatiquement le 19 avril 2019.

Trema 


\title{
Lecture et langage en français langue seconde : problèmes cognitifs et didactiques
}

\author{
Michèle Verdelhan-Bourgade
}

1 Dire que le débat sur la lecture est au centre du débat sur l'école est un cliché : déclarations ministérielles, rapports de l'Inspection générale, études d'experts, expériences scientifiques se croisent et s'entremêlent, parfois se contredisant pour alimenter un dossier sans cesse ouvert.

2 Un des aspects de ce dossier est la controverse actuelle sur la nature de l'acte de lire et des processus d'apprentissage. Elle est menée par des psychologues cognitivistes regroupés en France autour de Régine Kolinsky, Michel Fayol ou Jean-Emile Gombert par exemple, contre les travaux antérieurs d'autres psychologues comme E. Smith ou K.S. Goodman. Ce n'est pas seulement une querelle scientifique d'écoles en psychologie: les conséquences didactiques en sont énormes puisqu'il s'agit de prôner un enseignement de la lecture plus ou moins (voire complètement ou pas du tout) axé sur la mise en relation phonème - graphème. L'enjeu en est bien montré dans le récent ouvrage de José Morais, L'art de lire, paru fin 1994.

3 Dans le cadre de cet article on ne s'attachera pas à la discussion des thèses des uns et des autres, ni même à leur confrontation. On s'efforcera seulement de croiser ces débats actuels sur la lecture et la réflexion sur le français langue seconde qui fait l'objet de ce numéro. Quelles retombées ce débat peut-il avoir sur l'enseignement du français langue seconde ? Plus précisément, permet-il de mieux analyser les processus à l'œuvre lors de l'apprentissage de la lecture dans ce cadre et peut-on en tirer quelques conséquences didactiques, notamment en ce qui concerne les rapports de l'enseignement du langage et celui de la lecture? 


\section{Quelques bases de travail}

4 Sans prétendre faire ici un travail précis d'épistémologie, il est cependant souhaitable de préciser sur quelles conceptions des « objets intellectuels » à étudier on va se baser, à savoir le français langue seconde et la lecture.

5 Le français langue seconde est une notion éclairante mais complexe, les articles de J.-P. Cuq et $\mathrm{B}$. Maurer dans ce numéro le montrent bien. Elle recouvre des situations très diverses, en France et à l'étranger, dont nous retiendrons ici principalement deux aspects, en nous limitant au cas des enfants : celui des pays d'Afrique francophone et celui de la situation des immigrés en France. En Afrique francophone, les enfants, qui ont généralement appris à parler dans une langue 1, sont scolarisés vers 6-7 ans et apprennent alors à parler, lire et écrire en langue 2, le français, langue officielle, nationale ou administrative. En France, les enfants dits « rejoignants », arrivant de pays étrangers, parlant une langue 1, parfois la lisant ou l'écrivant, sont intégrés à l'école française où ils ont à apprendre à parler, lire écrire en français. Les deux situations ne sont pas identiques et comportent même de profondes différences ; elles présentent peutêtre aussi cependant des points communs en ce qui concerne les compétences que les enfants doivent mettre en jeu. Ce sera un des objets de cette réflexion.

Quant à la lecture, sans entrer là aussi dans les discussions du temps, on se basera simplement sur une affirmation d'il y a quelques années, dont le bon sens ne semble pas avoir été remis en question, à savoir qu'apprendre à lire c'est continuer à apprendre à parler, ou, sous une autre forme, que « lire est une activité langagière » (J. Hébrard, 1977, p. 57). On peut développer cette idée en y intégrant des travaux récents et définir l'acte de lire comme une activité cognitive (J. Morais, 1994,) portant sur du langage écrit. Ceci suppose que l'activité cognitive soit analysée en termes de processus cognitifs, mais aussi que le langage écrit soit analysé en termes linguistiques : composante phonologique de conversion de phonèmes en graphèmes, composante lexicale, composante syntaxique, composante discursive, sens, fonctions sociales et interactionnelles. Pour résumer, lire, ici, c'est lire des mots faits de graphèmes, des phrases, des discours, le tout ayant du sens et une fonction dans le groupe social.

\section{Apprentissage du lire et conscience phonémique}

7 De nombreux travaux sur les processus à l'œuvre dans la lecture posent l'existence d'une conscience "phonologique » ou "phonémique» dont on va rappeler rapidement le fonctionnement.

Cette notion renvoie à celles de «médiation phonologique » et de « lexique mental». Si l'on se réfère à R. Peereman (in R. Kolinsky, 1991), «chacun des mots que nous connaissons est représenté en mémoire dans une sorte de dictionnaire ou lexique mental ». A ce lexique interne conduisent deux voies d'accès: la voie orthographique directe, des lettres aux mots visuels, et la voie audio phonétique ou indirecte, transformation des lettres en sons. Et il existerait des "processus de conversion graphophonologique capables de générer une représentation phonologique à partir d'une séquence de lettres écrites. » (R. Peereman, p. 120). 
Les deux voies semblent coexister chez le lecteur normalement habile. La voie visuelle directe permet notamment de lire les mots familiers et de reconnaître les homonymes, du type «ceint, sain, saint». La voie phonologique permet, elle, de lire les mots inconnus, nouveaux, et les non-mots, du type « corfagron licromoux ... » (J. Morais, 1994, p. 158). Dans l'apprentissage, par contre, qui consiste en l'identification d'un grand nombre de mots nouveaux, la voie prépondérante serait la voie phonologique du décodage, qui convertit les signes graphiques en phonèmes selon des modèles qu'il convient à l'enfant d'intégrer.

La connaissance et l'usage de ces patrons de décodage s'appuient-ils sur une conscience phonémique antérieure? Pour J. Morais, qui reprend les travaux de J.E. Gombert (1990), les observations faites sur les adultes illettrés montrent que ceux-ci ne savent pas distinguer les phonèmes ; la conscience du phonème apparaîtrait en même temps que la connaissance du code alphabétique, l'une et l'autre se renforçant mutuellement (J. Morais, p. 185). La capacité métaphonologique serait donc contemporaine des apprentissages scolaires. Cependant, les travaux sur l'acquisition du langage, depuis R. Jakobson, se basent sur l'hypothèse d'une évolution par accès à la représentation phonémique de la langue : c'est parce que le bébé comprend qu'à un certain ensemble de sons correspond une signification, toujours la même, qu'il peut passer du babil au langage. Ensuite l'évolution se fera par différenciations successives à partir des voyelles et des consonnes de base : «mama » n'a pas le même sens que "papa, dada, caca » etc. Avant les premiers entraînements extérieurs à la différenciation des phonèmes, menés en France à l'école maternelle, et quelquefois dans la famille, l'enfant possèderait donc, sinon une conscience phonémique, du moins une expérience langagière du phonème, non explicitée mais réelle, sans laquelle il n'aurait pas appris à parler. Peut-être peut-on parler ici de pré-conscience phonémique.

11 Si la conscience phonologique ou phonémique est, comme l'affirment L. SprengerCharolles ou J. Morais, indispensable dans l'apprentissage de la conversion graphophonologique, elle-même indispensable dans l'apprentissage de la lecture, il faut aussi admettre que cette conscience ne naît pas du néant et s'appuie sur l'expérience langagière de l'enfant, expérience phonémique pré-consciente pourrait-on dire.

\section{Conscience linguistique et sociolinguistique}

L'écho rencontré par les travaux des cognitivistes sur la lecture, principalement centrés sur les modalités d'accès au lexique interne et donc à la voie graphophonologique, ne doivent pas faire oublier en effet qu'apprendre à lire est une activité complexe, portant sur un écrit lui-même complexe. La conscience phonémique est à nos yeux un élément dans un ensemble plus vaste, qu'on pourrait appeler "conscience linguistique», en référence au concept proposé par J. Downing et J. Fijalkow (1984). J. Downing a établi l'importance de la "clarté cognitive» dans les apprentissages, c'est-à-dire de la connaissance de ce qu'on fait, des objectifs de l'activité, des moyens à mettre en œuvre, de l'objet sur lequel on travaille. Ceci pourrait se traduire, en ce qui concerne l'apprentissage de la lecture, par la nécessité pour l'apprenant d'une conscience de l'écrit sous ses divers aspects: graphophonologique certes, mais aussi lexical, syntaxique, sémantique, sociolinguistique, psycholinguistique. 
13 L'écrit est du langage : cette évidence ne semble pas partagée par tous les apprentislecteurs, puisque certains tests sur des lecteurs maladroits font apparaître que ceux-ci n'ont pas compris que le texte écrit transcrivait du langage oral (E. Ferreiro, 1987). Ceci semble être une des causes fréquentes d'échec en lecture (G. Chauveau, E. RogovasChauveau, 1989).

14 L'écrit-langage obéit à des règles structurelles: la division en phrases, mots et syllabes, l'organisation des éléments dans l'énoncé. Apparemment ces principes sont les mêmes pour l'écrit et l'oral, et une conception de l'écrit comme codage de l'oral contribue à cette idée. Toutefois, la comparaison des unités de l'oral et de l'écrit, ainsi que de leurs formes d'assemblage, conduit à penser qu'il n'y a pas coïncidence exacte : la syllabe écrite ne se superpose pas à la syllabe orale, le mot obtient de l'écrit une forte légitimité liée à sa réalité visuelle, alors qu'il est bien plus difficilement identifiable à l'oral, la phrase est une réalisation écrite rarement atteinte dans l'oral quotidien... Acquérir une conscience linguistique de l'écrit suppose sans doute la compréhension que l'écrit est fait d'énoncés dont le sens pourrait aussi être transmis par l'oral ; que ces énoncés sont faits d'unités qui se combinent entre elles selon des modèles syntaxiques, que ces unités, syntagmes et mots, existent déjà dans l'oral.

On pourrait dire de la conscience linguistique ce qu'on a dit de la conscience phonémique. Avant le travail scolaire proprement dit, l'enfant a de la langue une expérience lexicale et syntaxique non explicitée. Il connaît des mots, il forme des phrases, sans forcément savoir ce qu'est un mot ou une phrase. C'est cette expérience pourtant qui va lui permettre de mettre de l'ordre dans le magma écrit et d'y attribuer de la signification. Les travaux actuels (Gombert, in Gombert-Fayol et coll. 1992) commencent à s'intéresser au rôle du traitement syntaxique des énoncés, le « calcul syntaxique de la signification ».

L'écrit-langage, enfin, obéit à des fonctions, dont la connaissance semble capitale dans l'apprentissage, et qui ne sont pas identiques à celles de l'oral.

17 M.A.K. Halliday (1974) a analysé chez les jeunes enfants l'évolution des fonctions du langage en trois phases, caractérisée par une réduction progressive, de six à trois, du nombre de ces fonctions. Il en arriverait ainsi à trois fonctions dominantes : une fonction idéationnelle de représentation du monde, une fonction interpersonnelle de mise en jeu des relations, et une fonction textuelle d'organisation interne du discours. Le haut degré de généralité de cette analyse lui permet de s'appliquer à la communication enfantine quel que soit le contexte culturel. Toutefois, il n'est pas sûr que d'autres fonctions n'apparaissent pas dans certains groupes ni que ces trois fonctions suffisent à rendre compte de la communication écrite, par exemple du rôle de trace joué par l'écrit, ou de celui de mise à distance de l'autre, ou encore de celui, occasionnel, de communication tournée vers soi.

18 Mais enfin apprendre à lire suppose bien la compréhension de ces fonctions langagières et la connaissance des usages sociaux des écrits; sinon à quoi bon apprendre? Cela suppose aussi une connaissance préalable du thème traité dans l'écrit (Gaonac'h, 1995), une familiarisation avec les modes scolaires du langage oral et écrit (formulation des ordres, consignes, tâches à accomplir...) et leur traduction matérielle dans les manuels, documents, exercices. La conscience linguistique s'accroît donc d'une conscience sociolinguistique de l'écrit. 


\section{Situations d'apprentissage en français langue seconde}

19 Essayons d'analyser l'apprentissage du français langue seconde d'après les paramètres énoncés précédemment, en examinant successivement le cas des enfants d'Afrique francophone et celui des enfants immigrés en France.

\subsection{Apprendre à lire en Afrique francophone}

A son arrivée à six ans à l'école, l'enfant sait parler dans une langue 1, langue maternelle, langue de la famille, du groupe social ou ethnique. Cette langue peut avoir un statut de langue officielle et une représentation écrite : c'est par exemple le cas du wolof, du sérère et du poular au Sénégal (Dumont, Maurer, 1995). Dans la plupart des cas cependant il s'agit d'une langue purement orale, souvent parlée par un groupe restreint. Il est fréquent que dans sa classe, nombreuse, le maître se trouve face à des enfants de langues maternelles très diverses.

21 L'enfant va devoir apprendre à parler, lire et écrire en français dès la première année d'école, c'est-à-dire être alphabétisé dans une langue différente de sa langue parlée. En termes de conscience linguistique ou phonémique, quelle est sa situation?

Parlant la langue 1, mais n'ayant pas eu de formation scolaire dans cette langue, on peut penser qu'il a une expérience pratique du phonème, mais non une conscience explicite. La conscience phonémique reste à acquérir par la pratique scolaire de la décomposition du mot en phonèmes ou du repérage des similitudes sonores. Mais l'enfant va devoir acquérir cette conscience sur une langue 2 , dont les phonèmes sont différents de ceux de la langue 1 par leur nombre, leurs caractéristiques sonores et les relations qu'ils entretiennent au sein du système phonique de la langue. Contrairement aux enfants français, l'enfant africain ne peut pas s'appuyer complètement sur son expérience langagière antérieure pour accéder à la conscience phonique en français.

De la même manière, il possède une pré-conscience linguistique en langue 1 qui lui permet d'agencer ses énoncés et de les adapter aux contraintes de la communication. Mais la nature des unités lexicales, sémantiques et syntaxiques, ainsi que leurs règles d'organisation, ne sont pas les mêmes en langue 1 et en français. Là encore, même s'il possède des connaissances implicites sur le langage, l'enfant ne peut guère tirer parti de son expérience linguistique pour développer la conscience linguistique indispensable à l'apprentissage. Certes, s'agissant d'une langue seconde et non étrangère, le français est parlé dans le pays, comme langue officielle ou de communication entre groupes. Ce français a d'ailleurs intégré des africanismes comme les langues africaines ont intégré des mots français (P. Dumont, B. Maurer, 1995). Mais le degré de familiarité linguistique avec le français est très divers selon l'origine géographique, sociale et familiale de l'enfant et il peut même être proche de zéro.

24 L'enfant qui possède une compétence de communication en langue 1 va donc l'étendre par l'apprentissage du français. A ceci près que les usages sociaux du langage ne sont pas les mêmes dans les deux langues, non plus que les règles de communication. De plus, la pratique de la langue 1 ne s'accompagne pas forcément d'une familiarisation avec l'écrit, puisque cette langue est généralement purement orale. L'écrit n'a alors pas de réalité 
sociale dans la langue maternelle, ses fonctions ne sont pas connues. Dans les villes où circulent des journaux, où l'on voit des affiches et des enseignes, l'enfant peut comprendre à quoi sert l'écrit. Dans les villages ou même les petites villes, l'écrit en français n'a pas de fonction apparente.

\subsection{Apprendre à lire en France pour les « rejoignants »}

On s'intéressera ici aux enfants étrangers nouvellement arrivés en France. Ils peuvent avoir été scolarisés et alphabétisés dans leur pays d'origine ou pas. Dans le premier cas, ils ont à transférer des compétences de lecteur d'un objet langue 1 à un objet langue 2. La conscience phonémique a dû être activée lors de l'apprentissage, la mise en relation des unités de l'oral et de l'écrit ayant été faite. De même pour les capacités méta linguistiques en général : la connaissance capitale qu'un énoncé est fait d'éléments qui se combinent selon certaines règles est déjà un savoir acquis.

Dans le deuxième cas, celui des enfants non alphabétisés, on retrouve des similitudes avec les enfants africains, tant sur le plan de la conscience phonémique que de la conscience linguistique. La principale différence consiste surtout dans le rôle et le statut de la langue dite seconde, le français ici étant la langue de la communication sociale quotidienne, de proximité, et pas seulement celle de la communication officielle ou administrative. C'est celle de l'insertion dans la société et cette insertion s'accompagne de l'apprentissage des règles françaises de communication. Les écrits abondent, à l'école et dans la rue, même s'ils ne sont pas toujours présents à l'intérieur de la famille, leur rôle peut donc être perçu et ce qu'en dit le maître n'apparaît pas comme une pure abstraction.

\section{Conséquences didactiques : la priorité au langage}

27 Si l'on essaie de récapituler les différentes remarques faites ci-dessus, on aboutit au raisonnement suivant.

- Si l'écriture française est alphabétique, cela veut dire qu'elle est une mise en relation de phonèmes et de graphèmes.

- Si la clarté cognitive est indispensable à l'apprentissage, alors l'apprentissage de la lecture nécessite entre autres le développement de la conscience linguistique, incluant celui de la conscience phonémique, ou plus généralement, de capacités métalinguistiques.

- Si l'émergence de cette conscience linguistique se fait par les pratiques scolaires d'analyse, mais en s'appuyant sur l'expérience langagière intériorisée par les enfants, alors il est indispensable de donner le temps et les soins nécessaires au développement de cette compétence.

Cela signifie concrètement qu'il faut donner à l'enfant qui apprend le français l'expérience langagière qui lui manque dans cette langue avant de chercher à lui apprendre à lire, comme c'est le cas actuellement le plus souvent. La situation d'enfant apprenant en français langue seconde est la plus difficile qui soit. A la différence de l'enfant dont la langue maternelle est le français, il n'a pas la pré-conscience linguistique née de la pratique préalable. A la différence de l'apprenant déjà scolarisé et qui aborde le français comme première ou deuxième langue étrangère, il n'a pas commencé à développer des compétences sociales et cognitives de lecteur, ni à réaliser la mise en relation oral-écrit. Or tous les travaux récents sur la lecture le montrent, la 
compréhension de l'écrit s'appuie sur les connaissances générales qu'a l'enfant sur le langage.

La logique des concepts de clarté cognitive, de conscience linguistique ou de capacités métalinguistiques aboutit à prôner un renouvellement dans l'articulation de l'enseignement $d u$ langage et de la lecture en français langue seconde. Mener systématiquement les deux de front dès le début revient sans doute à placer les enfants concernés dans une situation d'extrême difficulté dont les résultats, en particulier en Afrique francophone, se montrent d'ailleurs fort peu satisfaisants. L'apprentissage de la lecture gagnerait à être précédé d'une phase importante de pratique du langage en français, accompagnée progressivement de quelques activités destinées à la prise de conscience des réalités linguistiques de cette langue : phrases, mots, phonèmes. A cette activité langagière et linguistique s'ajouterait une familiarisation avec les fonctions de l'écrit, ses usages sociaux dans le groupe concerné. C'est seulement quand cette pratique serait assurée et la conscience linguistique ainsi amorcée qu'on devrait envisager l'apprentissage proprement dit de la lecture.

Les contraintes des institutions, les politiques linguistiques et scolaires, pèsent bien lourd face à des réflexions de chercheurs. Mais les difficultés de l'enseignement du français en Afrique francophone, ainsi que celles rencontrées par les enfants étrangers nouvellement arrivés en France, incitent à reposer la question de la chronologie des apprentissages, fûtce au prix de quelques déchirantes révisions.

\section{BIBLIOGRAPHIE}

CHAUVEAU G., ROGOVAS-CHAUVEAU E., « Les processus d'acquisition ou d'échec en lecture au cours préparatoire ", in Apprendre à lire et à écrire. Revue française de pédagogie, $\mathrm{n}^{\circ}$ spécial. Paris. INRP, 1989.

DOWNING J., FIJALKOW J., Lire et raisonner. Toulouse, Privat, 1984.

DUMONT P., MAURER B., Sociolinguistique du français en Afrique francophone. Paris. EDICEF AUPELF, 1995.

FAYOL M., GOMBERT J.-E., et coll., Psychologie cognitive de la lecture. Paris, PUF, 1992.

FERREIRO E., « Processus d'acquisition de la langue écrite dans le système scolaire », in Dossiers de l'éducation. 11-12, 15-29.

GAONAC'H D., Acquisition et utilisation d'une langue étrangère. Paris, Hachette, 1990.

GAONAC'H D. et GOLDER C. (coord.). Manuel de psychologie pour l'enseignement. Paris, Hachette, 1995.

GOMBERT J.-E., Le développement métalinguistique. Paris, PUF, 1990.

HALLIDAY M.A.K., « Dialogue with Herman Parret » in PARRET H. Discussing Language, La Haye, Mouton, 1974.

HEBRARD J., LENTIN, CLESSE C., JAN I., Du parler au lire. Paris, ESF, 1977. 
KOLINSKY R., MORAIS J., SEGUI J., La reconnaissance des mots dans les différentes modalités sensorielles : études de psychologie cognitive. Paris, PUF, 1991.

MORAIS J., L'art de lire. Paris, Odile Jacob, 1994.

PEEREMAN R., « La médiation phonologique dans la reconnaissance des mots écrits ». In Kolinsky et al. Paris, PUF, 1991.

SPRENGER-CHAROLLES L, «L'apprentissage de la lecture et ses difficultés », in Apprendre à lire et à écrire. Revue française de pédagogie, $\mathrm{n}^{\circ}$ spécial. Paris, INRP, 1989.

\section{RÉSUMÉS}

S'appuyant sur les travaux menés actuellement sur la lecture par la psychologie cognitive, l'auteur en étudie quelques implications didactiques dans l'enseignement du français langue seconde. Les concepts de conscience phonémique et de conscience linguistique, ainsi que les études sur la compréhension langagière amènent à poser la nécessité d'un apprentissage du langage oral préalable à celui de la lecture.

On the basis of the research work currently being conducted on reading by those who abide by the tenets of cognitive psychology, the author studies a few of the didactic implications of that research upon the teaching of french as a second language. The concepts of phonemic and linguistic consciousness, as well as studies on language comprehension lead to positing the necessity of the learning of oral language prior to that of reading.

\section{INDEX}

Mots-clés : conscience linguistique, français langue seconde (FLS), langage, lecture

Keywords : french as a second language (FSL), language, linguistic consciousness, reading

\section{AUTEUR}

\section{MICHÈLE VERDELHAN-BOURGADE}

Professeur en sciences du langage, IUFM et université Paul Valéry de Montpellier 